\title{
Prediction of melting point of the co-crystals: ANN models
}

$\underline{\text { Rama Krishna Rao Gamidi }}{ }^{1}$, Marko Ukrainczyk ${ }^{1}$, Jacek Zeglinski ${ }^{1}$, Åke C. Rasmuson ${ }^{1}$

${ }^{1}$ Department Of Chemical Science, Bernal Institute, University Of Limerick, Limerick, Ireland

E-mail: rama.gamidi@ul.ie

Using Artificial Neural Networks (ANNs), 1 a model has been developed for prediction of the melting point (Tm)2 of unsynthesized co-crystals (CCS). The model uses four input parameters for the pure Active Pharmaceutical Ingredient (APIs) and four for the pure coformer. In addition, as input parameters the model uses the 1:1 gas phase binding energy in the anticipated main synthon of the cocrystal as calculated by a force field method and $\Delta \mathrm{pKa}$ value of the respective cocrystals 2 (mostly extracted from the literature). The model is trained using known cocrystal melting temperatures giving an average relative deviation of $1.98 \%$, and can then predict the melting point of a validation set to a relative deviation of $3.37 \%$. In total, $61 \mathrm{CCs}$ (two-component molecular cocrystals3) Tm were used to construct the model, and the Tm values were extracted from the literature for four APIs, namely, i.e. caffeine (CAF), theophylline (THP), nicotinamide (NA) and isonicotinamide (INA). The number of CCs included were: 14-CAF, 9-THP, 29-INA and 9-NA. The advantage of our model is that, it could be possible to set the melting point of the new solid form of the respective drug molecules within the target range by a selection of an ideal coformer for cocrystal formation. Hence, it will reduce the cost, manpower and time in the pharmaceutical industry.

[1] Svetlana. I. et. al. (2012), Pharmaceutics. 4, 531-550.

[2] Gamidi, R. K. et al. (2017), Cryst. Growth Des. 17, 175-182.

[3] Duggirala, N. K. et al. (2016), Chem. Commun. 52, 640-655.

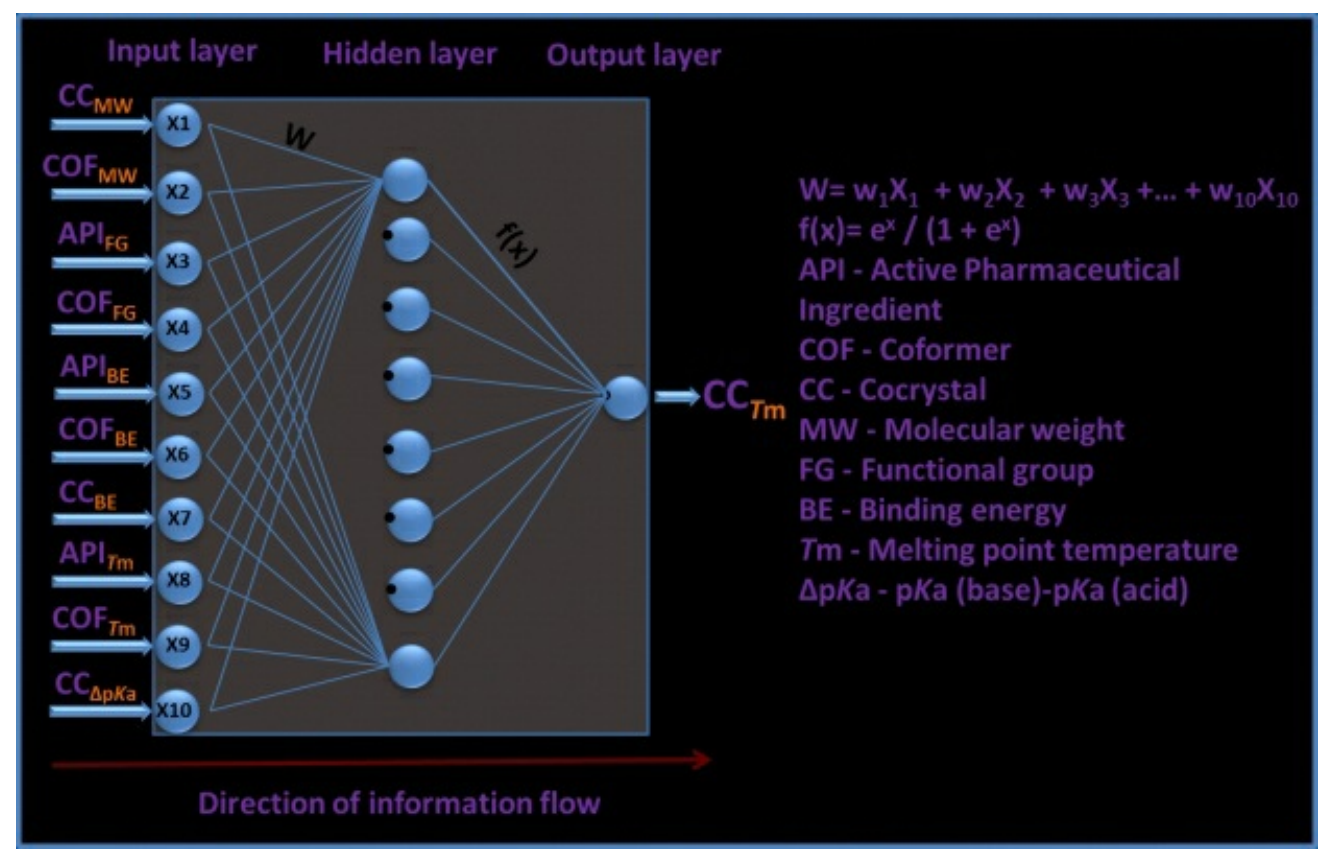

Keywords: Co-crystals, Melting point, ANN models 